\title{
Guidelines on the Ethics of Clinical Research in Anesthesia
}

\section{.}

1 HESE guidelines were prepared by the Committee on Bioethics of the Canadian Anesthesiologists' Society to assist investigators in consideration of the ethical issues involved in anesthesia research with human subjects. These guidelines cannot be an exhaustive treatment of the subject, nor advise on every study design. Investigators are urged to use these guidelines, to discuss the relevant issues with local ethics consultants and use the resources of local Research Ethics Boards in keeping with the Tri-council Policy Statement, Ethical Conduct for Research Involving Humans.

1. Joint publication of the Medical Research Council of Canada, the Natural Sciences and Engineering Research Council of Canada, and the Social Sciences and Humanities Research Council of Canada, August 1998

2. While obtaining consent from a legally responsible or legally authorized representative is accepted in the context of therapy, it is still not without controversy in the research context. The issue of recruiting potentially incapable persons in anesthesia research must be carefully considered by the local Research Ethics Board.

3. As an example, in some centres the research nurse is viewed as part of the departmental team and contacts potential research patients in advance to ask if they are interested in participating. This is accepted by the Research Ethics Board, is not viewed as invasion of privacy and is well received by prospective subjects.

4. Tri-Council Policy Statement, Ethical conduct for research involving humans, August 1998; 7.4.

5. Freedman B., Equipoise and the ethics of clinical research. N Eng J Med, 1987; 317(3): 141-5.

6. Weijer C. Can Med Assoc J 1997; 157: 1020.

Preamble

As part of its mandate to promote high-quality research, the Canadian Anesthesiologists' Society rec- ognizes the need to ensure appropriate moral and ethical behaviour on the part of investigators who conduct clinical research with human subjects in Canada.

For human research, in anesthesia as in any other specialty, investigators are expected to comply with the Tri-Council Policy Statement, Ethical Conduct for Research Involving Humans.1 which uses a principlebased framework. Its "cardinal principle", is respect for human dignity. Ethical principles such as respect for free and informed consent, respect for privacy and confidentiality, beneficence (maximizing benefits), nonmaleficence (minimizing harm), and respect for justice and inclusiveness must be carefully weighed in circumstances where they may be in conflict. Researchers may carry out research protocols only after approval is obtained from an appropriate institutional Research Ethics Board (REB). The REB should also be responsible for monitoring the progress of each study.

It is apparent that a number of specialty-related ethical dilemmas arise with certain research protocols. In the unique environment of anesthesia, these include factors such as adequate time for patients to reflect on the protocol, and their ability to withdraw at any time. In the latter context, it is recognized that clinical trials involving general anesthetics or other potent central nervous system depressant medications necessarily limit the patient's ability to withdraw from the study at some times. Another example of challenges inherent in anesthesia trials is the issue of determining how, when and by whom patients should be approached for consent. Because of such ethical dilemmas, the Canadian Anesthesiologists' Society supports the thoughtful interpretation and application of existing guidelines and ethical principles to each human research protocol.

\section{Research with Human Subjects}

\section{A. Informed Consent}

Ethical conduct of research requires that informed consent be obtained from participating subjects, or 
from an appropriate legally responsible delegate. 2 Information regarding the study must be explained to prospective subjects or participants in lay terms, and must include providing the protocol information in writing. The consent form must also state that the individual may refuse to participate, or is free to withdraw from participation, at any time without prejudice to his or her medical care. (See Appendix: Sample Consent Form).

Timing of Obtaining Informed Consent for Research The amount of time required for patients to make an informed decision about participating in research is an important issue. A problem may arise in anesthesia because anesthesia investigators may not have their first contact with patients until they are in hospital, often on the same day as their scheduled surgical procedure. If the investigator is not the patient's anesthesiologist, an ethical conflict may arise. If prior contact with potential study subjects is required by the Research Ethics Board, such contact by a "stranger" either at work or at home, could be perceived by the patient as a breach of confidentiality and invasion of privacy. Conversely, if the investigator will also be the patient's clinical anesthesiologist there is a duty-ofcare relationship, and such an approach might be considered coercive. The balance between competing ethical considerations must be carefully evaluated for each research protocol.3

The Canadian Anesthesiologists' Society believes that pre-op erative consent for clinical research in anesthesia may be obtained after admission to hospital, either before or on the day of the scheduled surgery provided that:

1. patients are not under the influence of premedication.

2. risk to the patient and time commitment to the study are not significantly different from routine clinical care (eg during drug trials in which a patient may be randomly to receive one of several anesthetic regimens, using approved medications).

3. after verbal explanation from the investigator or research assistant and in the absence of the investigator or research assistant, patients are given time to read the information sheet and consider the risks and benefits. Patients should have an opportunity to raise any further questions or seek clarification on any points concerning the nature of the study, alternatives, risks, benefits, etc.

4. patients who feel they are under duress, or require more time to make a decision, should be advised to decline participation in the study.
5. investigators document in the health care chart the nature of their consent process for patients who agree to participate in a research protocol.

For protocols that require patients to receive advance information because of additional risk to or time commitment from the patient or for administrative reasons connected with the protocol, the principal investigator and the REB should agree upon an ethical and practical mechanism to provide this information.

\section{B. Privacy and Patient Confidentiality}

Privacy involves the right of the study participant to decide the extent to which there is access to personal data that is not already in the public domain. Confidentiality involves the preservation of the subject's anonymity when handling data during research and during its subsequent use in teaching, scholarly presentations, and in publication. As with any clinical research, privacy and confidentiality must, at all times, be respected. In this regard, the nature and type of information that is accessed for study purposes should be documented, and if that information is to be used for other reasons, subjects should be made aware of this as part of the consent process.

\section{The Potential for Risks and Benefits}

The nature of many clinical trials in anesthesia (eg, comparison of the recovery characteristics and cost/benefit ratios of different types of general anesthetics) is such that the primary risk is that of the anesthetic itself, not participation in the study protocol. On the other hand, use of invasive monitoring techniques to evaluate cardiovascular effects of a new anesthetic agent may involve the risk of rare but potentially serious complications. It is always the responsibility of the investigator and the REB to ensure that projected benefits outweigh the possible risks.

When consenting to participation in research, subjects accept the possibility of risks and benefits. The probability of risk and the magnitude and character of potential harm must be disclosed. (eg, minimal risk of minimal harm, or minimal risk of significant harm). The likelihood of a given risk, its duration and likely reversibility must be assessed. Investigators must also be prepared to demonstrate that there is no reasonable alternative methodology that would avoid or reduce possible risks. For some studies, consideration should be given to the use of healthy volunteers instead of patients.

Benefits may include potential advantages to the subject, future patients, third parties, society or a segment thereof, and any general increase in human knowledge. 


\section{Placebos}

Use of a placebo medication as the control arm in an anesthesia protocol is unethical when there is an established effective treatment for the condition under investigation. "The use of placebo controls in clinical trials is generally unacceptable when standard therapies or interventions are available for a particular patient population." 4

This is based on the concept of clinical equipoise, which exists only when there is genuine uncertainty among experts about the relative therapeutic merits of the two arms of a clinical trial. It is expected that a clinical trial will be designed so that, if successful, it will provide evidence in favour of one of the treatments. 5

For ethical use of a placebo control there must be genuine disagreement among expert practitioners as to preferred treatment. "A nonvalidated treatment may be compared with a placebo control if ( 1 ) no standard therapy exists, (2) standard therapy exists but has been shown to be no better than placebo, (3) standard therapy is placebo, (4) standard therapy is toxic and of marginal benefit, or, (5) validated treatment exists but is not available because of cost or limited supplies.... Placebo controls are appropriately used when the new, nonvalidated treatment is an 'add-on' to standard therapy (so that the comparison is standard therapy plus new drug versus standard therapy plus placebo)."6

\section{E. Conflicts of Interest}

All potential conflicts of interest, must be declared verbally to participants in a clinical trial and in writing in the consent form and any subsequent publications. Financial or material rewards to investigators must also be declared to the Research Ethics Board.

Clinical care must always take precedence over research investigation. When the same anesthesiologist will be both investigating research subjects and providing their anesthetic care, the research protocol as approved by the Research Ethics Board must have clear indications as to when the study protocol will be abandoned for the well-being or safety of the individual patient.

\section{F. Remuneration to Study Participants}

Research subjects must not be induced by promises of reward to take risks in research that they would not otherwise undertake. Equally, research subjects must not be expected to subsidize research by suffering monetary or other losses. With the approval of the Research Ethics Board subjects' expenses may be reimbursed.

\section{G. Vulnerable Populations}

These populations include patients who lack decisionmaking capacity, patients of questionable capacity, and those who may be "capable" but vulnerable because of a disease process, culture, or inability to speak a language. It is unethical to take advantage of such populations in recruiting participants to a clinical study. In general, capable people should be enrolled where possible over those who are incapable or of questionable capacity. Research protocols must use appropriate models and populations, taking into consideration issues of gender and age. In particular, it is important that there be on-going research in anesthesia for the pediatric population as it would be unethical to deny this age group the benefits of advances in the specialty. Investigators and research ethics boards have a particular duty to protect child participants and those from other vulnerable populations from undue risk of harm, especially when consent or permission is obtained through another person.

Competence to consent to research does not require that patients be competent in all respects for all purposes. Decision making capacity is not a global assessment but a functional assessment and is decision specific. For example, a patient who is incapable of managing financial affairs may be competent to consent to research.

In conducting research that might involve cognitively impaired persons, the protocol must include an assessment of competence. Furthermore, a person who is incompetent, or of doubtful competence, must not be included in research that poses more than minor risks without substantial benefits for that person. In addition, if incompetent persons are to be enrolled, the researcher must explain to the REB how third party authorization will be obtained, and that the subject's interests are protected.

\section{Major Sources Consulted}

1. Guidelines for Research Involving Humans. Medical Research Council of Canada. 1987.

2. World Medical Association Declaration of Helsinki: Recommendations Guiding Medical Doctors Involving Human Subjects. 1989.

3. Same Day Consent for Anesthesia Research. Can J Anaesth 1994; 41:1234.

4. Manuel sur le soin et l'utilisation des animaux d'expérimentation. Conseil canadien de protection des animaux. Vol 1, 1980; Vol 2; 1984.

5. Animals in Biomedical Research, CMA POLICY SUMMARY. Can Med Assoc J, 1986; 135. 
6. National Council on Bioethics in Human Research. Ethics of Research Involving Children. Communiqué. 1993; 4 (1)

7. National Council on Bioethics in Human Research. Communiqué. (Consent for Research in Anaesthesia.) 1993; 4 (1)

8. Human Tissues Gift Act. Federal Ministry of Health, Canada.

9. Guidelines for the Ethical Practice of Orthopaedic Surgery. Canadian Orthopaedic Association. 1995.

10. Tri-Council Policy Statement: Ethical Conduct for Research Involving Humans. The Medical Research Council of Canada; The Natural Sciences and Engineering Research Council of Canada; The Social Sciences and Humanities Research Council of Canada. August 1998.

\section{APPENDIX \\ SAMPLE CONSENT FORM \\ Research Project \\ Investigators \\ Funding Agency}

This consent form, a copy of which has been given to you, is only part of the process of informed consent. It should give you the basic idea of what the research project is about and what your participation will involve. If you would like more detail about something mentioned here, or information not included here, you should feel free to ask. Please take the time to read this carefully and to understand any accompanying information.

(The investigators should supply the following information in ordinary language, avoiding jargon and supplying explanations for crucial terms. This is a sample consent form, so if any item is obviously irrelevant it need not be included).

1. Purpose of the research.

2. Description of all experimental agents and procedures, including identification of those that would not be a part of routine therapeutic care.

3. Explanation of such aspects of research design as randomization and double-blinds. In the case of double-blinds, details of when a code may be broken, debriefing and final consent after debriefing should be included.

4. Description of the likelihood of any discomforts and inconveniences associated with participation and of known or suspected short- and long-term risks.

5. Detailed descriptions of exactly what participation will entail (regular routines, time commitment, natures of tests and procedures, etc.).
6. Probability and nature of direct and indirect benefits to the subjects themselves and to others.

7. Identification of alternatives to enrollment in the research, with discussion of the risks, benefits, etc. of these alternatives. (Where appropriate, this disclosure may be verbal or supplementary to the consent form and referred to at this point).

8. Explanation of who will have access to information collected and the identity of the subject, including a description of how confidentiality will be protected.

9. Explanation of how the subjects will receive new or updated information during the course of the research.

10. Description of any financial costs the subject may incur as a condition of or because of participation in the research.

Your signature on this form indicates that you have understood to your satisfaction the information regarding your participation in the research project and agree to participate as a subject. In no way does this waive your legal rights nor release the investigators, sponsors, or involved institutions from their legal and professional responsibilities. You are free to withdraw from the study at any time without jeopardizing your health care. Your continued participation should be as informed as your initial consent, so you should feel free to ask for clarification or new information throughout your participation. If you have further questions, please contact:

Name of responsible investigator or qualified designate

Phone Number

(Name)

(Signature of subject, or responsible proxy)

(Name of Witness)

(Signature of witness)

(Date) 